\title{
Processo de implantação do e-SUS Atenção Básica em Sobral - CE
}

\author{
The process of implementation of the e-SUS AB (primary health care) in \\ Sobral-CE
}
Proceso de implantación del e-SUS AB (atención primaria de salud) en Sobral-CE

Marcos Aguiar Ribeiro ${ }^{1, a}$

marcosaguiar61@hotmail.com | https://orcid.org/o0oo-0001-7299-8007

Thayná Biatriz Farias Muniz ${ }^{2, b}$

thaynabiatriizfm@hotmail.com | https://orcid.org/oooo-0oo1-8318-624X

Izabelle Mont 'Alverne Napoleão Albuquerque ${ }^{2, c}$

izabellemontalverne@gmail.com | https://orcid.org/o00o-0003-0856-5607

Aline Ávila Vasconcelos ${ }^{3, d}$

alineavila@hotmail.com | https://orcid.org/o0oo-0002-3047-4093

Mariana Moreira da Costa ${ }^{2, e}$

maryanacostafreire@hotmail.com | https://orcid.org/oooo-0003-2514-9736

Amanda Maria Braga Vasconcelos ${ }^{2, f}$

amandabragav@gmail.com | https://orcid.org/o00o-0003-4699-0233

\footnotetext{
${ }^{1}$ Universidade Federal de São Paulo. São Paulo, SP, Brasil

2 Universidade Estadual Vale do Acaraú. Sobral, CE, Brasil.

3 Universidade Estadual do Ceará. Fortaleza, CE, Brasil.

a Mestrado em Saúde da Família pela Universidade Federal do Ceará.

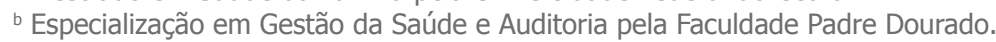

c Doutorado em Enfermagem pela Universidade Federal do Ceará.

d Especialista em Gestão da Saúde e Auditoria pela Faculdade Padre Dourado.

e Graduanda em Enfermagem pela Universidade Estadual Vale do Acaraú.

f Graduanda em Enfermagem pela Universidade Estadual Vale do Acaraú.
}

\section{Resumo}

Criado pelo Ministério da Saúde (MS), o e-SUS AB vem com a finalidade de reorganizar os dados da atenção básica e reestruturar os sistemas de informação em saúde. O objetivo do estudo que fundamenta este artigo foi conhecer os desafios da implantação do e-SUS Atenção Básica no programa Estratégia de Saúde da Família do município de Sobral - CE. Caracteriza-se como uma pesquisa exploratória e descritiva com abordagem qualitativa, realizada com seis equipes do programa Estratégia de Saúde da Família no município de Sobral por meio de entrevistas semiestruturadas aplicadas em trinta profissionais de saúde. Para a realização da análise de dados, foi utilizada a estratégia do discurso do sujeito coletivo. Os resultados da pesquisa evidenciaram que o e-SUS AB contribui positivamente para a gestão e a assistência no sistema de saúde, mas que existem diversos obstáculos para que o sistema tenha perfeita funcionalidade e concretização. Contudo, ainda existem aspectos restritivos para um funcionamento satisfatório dessa ferramenta.

Palavras-chave: e-SUS; Atenção primária à saúde; Sistemas de informação; Sistema Único de Saúde; Discurso do sujeito coletivo. 


\begin{abstract}
Created by the Ministry of Health (MS) of Brazil, the e-SUS AB has the purpose of reorganizing the data of primary health care and restructuring the health information systems. This article bases on a study that has aimed to know the challenges to carry out e-SUS AB on the programme Estratégia de Saúde da Família (family health strategy) which offers health care to the population of Sobral, CE, Brazil. It is characterized as an exploratory and descriptive research using a qualitative approach carried out in six teams of the programme Estratégia de Saúde da Família in Sobral through semi-structured interviews with thirty health professionals. For the data analysis the the discourse of the collective subject method was used. The results of the research evidenced that e-SUS AB contributes positively to the management and the assistance in the health system, but that there are different obstacles for the system to have perfect functionality and can materialize. However, there are still constraints to the satisfactory operation of this tool.
\end{abstract}

Keywords: e-SUS; Primary health care; Information systems; Unified Health System; Discourse of the collective subject.

\title{
Resumen
}

El e-SUS AB ha sido creado por el Ministerio de Salud (MS) de Brasil y viene con la finalidad de reorganizar los datos de la atención primaria de salud y reestructurar los sistemas de información en salud del MS. El objetivo del estudio que fundamenta este artículo fue conocer los desafíos de la implantación del e-SUS AB en el programa Estratégia de Saúde da Família (estrategia de salud de la familia) del municipio Sobral, CE, Brasil. El estudio se caracteriza como una investigación exploratoria y descriptiva con abordaje cualitativo centrada en seis equipos del programa Estrategias de Salud de la Familia en el municipio de Sobral por medio de entrevistas semiestructuradas realizadas con treinta profesionales de salud. Para el análisis de datos se utilizó la estrategia del discurso del sujeto colectivo. Los resultados de la investigación evidenciaron que el e-SUS AB contribuye positivamente al éxito de la gestión y de la asistencia en el sistema de salud, pero que existen diversos obstáculos para que el sistema tenga perfecta funcionalidad y concretización. Sin embargo, todavía existen aspectos restrictivos para el funcionamiento satisfactorio de esta herramienta.

Palabras clave: e-SUS; Atención primaria de salud; Sistemas de información; Sistema Único de Salud. Discurso del sujeto colectivo.

\section{Contribuição dos autores:}

Concepção e desenho do estudo: Marcos Aguiar Ribeiro e Thayná Biatriz Farias Muniz.

Aquisição, análise ou interpretação dos dados: Marcos Aguiar Ribeiro e Thayná Biatriz Farias Muniz.

Redação do manuscrito: Marcos Aguiar Ribeiro, Thayná Biatriz Farias Muniz, Mariana Moreira da Costa, Amanda Maria Braga Vasconcelos. Revisão crítica do conteúdo intelectual: Marcos Aguiar Ribeiro, Izabelle Mont'Alverne Napoleão Albuquerque e Aline Ávila Vasconcelos.

Declaração de conflito de interesses: Este trabalho não apresenta conflito de interesses.

Fontes de financiamento: não há.

Considerações éticas: $O$ estudo foi submetido ao Comitê de Ética e Pesquisa com Seres Humanos da Universidade Estadual Vale do Acaraú - UVA, número do parecer: 1.958.303, sendo aprovado pelo mesmo.

Agradecimento/Contribuições adicionais: Agradecemos ao Observatório de Pesquisas para o SUS pelo apoio no desenvolvimento do Projeto.

Histórico do artigo: Submetido: 14.ago.2017 | Aceito: 23.jul.2018 | Publicado: 25.set.2018.

Apresentação anterior: não houve.

Licença CC BY-NC atribuição não comercial. Com essa licença é permitido acessar, baixar (download), copiar, imprimir, compartilhar, reutilizar e distribuir os artigos, desde que para uso não comercial e com a citação da fonte, conferindo os devidos créditos de autoria e menção à Reciis. Nesses casos, nenhuma permissão é necessária por parte dos autores ou dos editores. 


\section{Introdução}

A $8^{\text {a }}$ Conferência Nacional de Saúde teve como tema estruturante a indispensabilidade de um novo sistema de saúde e consolidou um instrumento que veio intervir nos encargos do Estado de garantir o direito à saúde para toda a população assegurando condições de acesso e qualidade dos serviços e ações ${ }^{1}$.

Nessa perspectiva, o Sistema Único de Saúde (SUS) foi criado para reverter as iniquidades de saúde da população brasileira a partir de ações e serviços orientados pelos princípios doutrinários e organizativos: universalidade, integralidade, equidade, regionalização, hierarquização, descentralização e participação popular. Dessa forma, o SUS integra o conjunto das ações e serviços da União, estados, Distrito Federal e municípios, onde cada parte cumpre funções e competências especificas, porém articuladas entre si, o que caracteriza os níveis de gestão nas três esferas governamentais ${ }^{2-4}$.

O Departamento de Atenção Básica (DAB) tem suas funções definidas pelo Decreto 7.530/2011 e pela Portaria 2.488/2011 e, além disso, normatiza e coordena a implantação de políticas e programas estratégicos no âmbito do Ministério da Saúde, tais como: Estratégia Saúde da Família (ESF); Programa de Requalificação das Unidades Básicas de Saúde; Programa Nacional de Melhoria do Acesso e Qualidade na Atenção Básica (PMAQ-AB); Programa Telessaúde Brasil Redes; Equipes de Consultórios na Rua; Programa Saúde na Escola (PSE), entre outros ${ }^{5,6}$.

O enfermeiro assume um papel importante na identificação das necessidades de cuidado da população, bem como na promoção e proteção da saúde dos indivíduos em suas diferentes dimensões. O gerenciamento organizacional de enfermagemé, portanto, um componentefundamental no sistema de saúdelocal, que apresenta os seus reflexos nos níveis regional e nacional. Além desses aspectos, a atuação dos agentes comunitários de saúde (ACS) é destacada na formação de redes de relacionamentos que se caracterizam como significativos na vida das pessoas, incluído relações de família, amizade, trabalho, estudo e comunidade, e também agências sociais e institucionais. Deste modo, o trabalho próximo às comunidades pode contribuir para a articulação de redes sociais, de maneira a fortalecer as relações entre os usuários e os sistemas de cuidado7.

Nesse contexto, o Departamento de Atenção Básica do Ministério da Saúde criou o e-SUS Atenção Básica (e-SUS AB), que substitui o Sistema de informação da atenção básica (Siab) e tem a finalidade de reorganizar os dados da atenção básica. Esta prática está alinhada com a sugestão de reestruturação dos sistemas de informação em saúde do Ministério da Saúde, já que a melhora da gestão dos dados é crucial para aumentar a qualidade no atendimento à população. A estratégia e-SUS AB faz referência ao processo de informatização qualificada do SUS em busca de um SUS eletrônico. Ele será utilizado pelos profissionais de saúde das equipes das unidades básicas de saúde e, ao contrário dos programas que vigoravam antes ou que ainda vigoram em alguns locais, o e-SUS dá acesso a informações individuais de cada usuário. A partir disso, é feita a escolha da maneira de transmissão dos dados, ou seja, se serão transmitidos através de CDS (Coleta de dados simplificada) ou PEC (Prontuário eletrônico do cidadão), ou de algum outro sistema já adotado pela unidade ${ }^{8}$.

Dada a sua importância, têm-se como objetivo conhecer os desafios da implantação do e-SUS AB na Estratégia de Saúde da Família do município de Sobral - CE utilizando a técnica do discurso do sujeito coletivo (DSC).

\section{Estratégia metodológica}

O estudo aqui apresentado caracteriza-se como uma pesquisa exploratória e descritiva com abordagem qualitativa.

O campo de atuação correspondeu ao Sistema Municipal de Saúde de Sobral. O município de Sobral - CE dispõe de uma rede de atenção à saúde hierarquizada e regionalizada com serviços em diferentes níveis de complexidade, atuando como polo para a Macrorregião Norte do Ceará9. 
Em relação à atenção básica, Sobral tem 64 equipes de ESF, distribuídas em 36 unidades básicas de saúde (UBS). Para melhor organização e planejamento, a gestão do município distribui as UBS em macroáreas. Em Sobral, existem quatro macroáreas, e a macroárea três foi selecionada para a realização do estudo. Esta macroárea tem uma população total de 53.226 habitantes e é composta por UBS da sede do município, o que justificou a seleção intencional, uma vez que essa composição possibilita melhor acesso dos pesquisadores para o desenvolvimento do estudo. Vale salientar, ainda, que o sistema local de saúde tem seis equipes do Núcleo de Apoio à Saúde da Família (NASF), localizadas em áreas compreendidas como territórios, que dão cobertura assistencial a 98\% da população, o que equivalem a 214.206 pessoas acompanhadas 9 .

Participaram do estudo trinta profissionais atuantes na ESF, sendo seis enfermeiros gerentes, seis enfermeiros da assistência e 18 agentes comunitários de saúde (ACS). A escolha desses participantes é justificada pelo fato de esses profissionais serem os principais atores no processo de implantação do e-SUS AB.

Os participantes tinham de obedecer aos seguintes critérios: atuar na ESF; ter no mínimo seis meses de experiência na ESF, visto que se considera este período um tempo razoável para vivenciar o cotidiano na ESF, de forma a colaborar e dar subsídios para a pesquisa. Além disso, foram considerados critérios de exclusão: estar de férias, de licença médica ou afastado por qualquer natureza no período da coleta de dados.

A coleta de dados foi realizada por meio de um roteiro de entrevista semiestruturada. Os dados foram coletados no período de março a junho de 2017, seguindo rigorosamente as perguntas previamente estabelecidas.

Após a seleção dos participantes do estudo, de acordo com os critérios de inclusão, eles foram indagados sobre o melhor local para realização da entrevista, visando à sua necessidade e disponibilidade. A entrevista foi gravada conforme autorização de cada participante do estudo.

Para análise dos dados, foi utilizada a estratégia do discurso do sujeito coletivo (DSC), que é uma proposta de organização de dados qualitativos de cunho verbal, colhidos via depoimentos. Tem como fundamento a teoria da representação social e as deduções sociológicas. A ideia consiste basicamente em analisar o material verbal coletado, extraindo de cada depoimento as expressões-chave que correspondem às ideias relevantes e que são a síntese do conteúdo discursivo ${ }^{10}$.

Para tal, utilizou-se o programa QualiQuantiSoft versão 1.3 visando facilitar a utilização da técnica de análise de dados do DSC, proposta por Lefevre e Lefevre. O programa QualiQuantiSoft é um software desenvolvido pela Sales e Paschoal Informática em parceria com a Universidade de São Paulo (USP), por intermédio da Faculdade de Saúde Pública, representada pelos professores Fernando Lefevre e Ana Maria Cavalcanti Lefevre, criadores da metodologia do DSC.

Os participantes do estudo foram esclarecidos sobre os objetivos da pesquisa, de forma a autorizar sua participação a partir da assinatura do Termo de Consentimento Livre e Esclarecido (TCLE). A pesquisa foi orientada a partir da Resolução de $\mathrm{N}^{0}$ 466, de 12 de dezembro de 2012, do Conselho Nacional de Saúde, que incorpora, sob a ótica do indivíduo e das coletividades, os quatro referenciais básicos da bioética: autonomia, não maleficência, beneficência e justiça, entre outros, visando assegurar os direitos e deveres que dizem respeito aos participantes da pesquisa, à comunidade científica e ao Estado ${ }^{11}$.

\section{Resultados e discussão}

Para facilitar a visualização do universo simbólico, os resultados e a discussão têm como base as respostas dos participantes da pesquisa apresentadas na forma de DSC e organizadas por questão respondida.

Quando indagados a respeito da compreensão do e-SUS, surgiram visões divergentes entre os profissionais entrevistados. Desta forma, observou-se que as respostas viabilizaram a construção de dois DSC distintos (DSC A e DSC B). O DSC A foi construído a partir das contribuições de enfermeiros e ACS e 
tem como ideia central a compreensão conforme a orientação institucional do Departamento de Atenção Básica (DAB) - Ministério da Saúde. Ou seja, de acordo com eles:

Oe-SUS é uma estratégia do Departamento de Atenção Básica (DAB) para, por meio da informatização, veicular as informações em nível nacional. É um programa que veio para substituir o Siab e busca realmente sistematizar o serviço para organizar melhor e de maneira informatizada os dados da atenção básica. Trata-se de uma estratégia de cadastro da população nacional destinado a ser alimentado com os cadastros familiares e individuais da população adscrita nos territórios, cadastros estes realizados pelos agentes comunitários de saúde. (DSC A)

Ao observar e analisar esse discurso, é possível perceber que os profissionais (seis enfermeiros e oito ACS) conhecem e compreendem a proposta do e-SUS lançada pelo Departamento de Atenção Básica (DAB).

Esses profissionais entendem a essência desse sistema de informação (SI), sua importância e seus propósitos, fator que contribui para que possam sempre colaborar da melhor maneira possível, a fim de se alcançarem os objetivos que o Ministério da Saúde propõe e obter resolutividade.

O e-SUS AB faz parte da estratégia e-Saúde, que corresponde ao uso das tecnologias de informação e comunicação (TIC) em questões relacionadas à saúde e busca reestruturar e integrar as informações da Atenção Básica em nível nacional ${ }^{12}$.É uma estratégia de resolutividade, por meio da esfera virtual, no âmbito da saúde e garante um processo amplo e padronizado de troca de informações entre sistemas em vários níveis de atenção e no próprio nível da atenção básica ${ }^{13}$.

Além disso, alguns profissionais (nove enfermeiros e sete ACS) percebem ainda o e-SUS como um dispositivo com objetivo de conhecer o perfil epidemiológico, a fim de traçar metas com base nos dados obtidos. Evidencia-se que as respostas desses profissionais proporcionaram a construção do DSC B, que tem como ideia central a compreensão do e-SUS como ferramenta de coleta de dados para a organização e o planejamento. Conforme suas respostas:

É através desse processo que será possível traçar metas. Quando a gente vê dados reais nos cadastros, a gente sabe quantos hipertensos há, quantos você atendeu no mês, quem foi atendido, e essas informações são importantes para gerar relatórios e esboçar objetivos de acordo com a realidade de cada território. Ele é uma importante ferramenta para se estabelecer o diagnóstico de saúde e das condições de vida das comunidades, além de mostrar significativamente as ações a serem realizadas pela Estratégia Saúde da Família (ESF). (DSC B)

Diante do exposto, é perceptível que esses profissionais assimilam que, além de um sistema de informação para organizar e armazenar dados, o e-SUS, tem outros propósitos a curto e longo prazo, com uma visão intensificada e específica de cada área, em alusão às necessidades da população de acordo com os problemas identificados.

Os sistemas de informação de saúde (SIS) precisam informar sobre a doença dos indivíduos e o seu perfil na comunidade, sobre as suas causas e condições, condutas, tecnologias em saúde utilizadas, ações a serem desenvolvidas e resultados, como proporção e impacto das ações na população ou grupos de risco. Eles têm o intuito de processar, armazenar, coletar e propagar dados, de modo a facilitar a gestão e ocasionar o aprimoramento das ações por ela desenvolvidas, visto que são capazes de oferecer suporte ao processo determinante em saúde ${ }^{14-15}$.

Quando indagados acerca da incorporação do e-SUS nas unidades de saúde, verifica-se que as respostas de 18 profissionais (dez enfermeiros e oito ACS) proporcionaram a construção do DSC C, que reverbera 
a ideia central de implantação do e-SUS e reforça a preocupação do Ministério da Saúde em preparar os profissionais para lidarem com a nova realidade. Segundo eles:

Foi implantado por etapas e, logo no início, todos os profissionais foram capacitados pelos organizadores do sistema e rotineiramente pelos colegas de trabalho para passarem a entender o processo de implantação e qual seria o motivo da mudança. Toda a equipe recebeu treinamento, oferecido pela coordenação primária, de pessoas que já tinham se apropriado da documentação e vinham fazer o repasse para toda a equipe, dizendo o que era o e-SUS, como era feito, quais eram as planilhas que a gente ia ter que utilizar para que esse e-SUS viesse passar a valer, desde os enfermeiros até os digitadores. As capacitações ocorriam geralmente em oficinas semanais com um facilitador mostrando todas as fichas com as quais as categorias iriam trabalhar. (DSC C)

Uma das etapas que o Departamento de Atenção Básica sugere na implantação da estratégia é a capacitação das equipes de saúde e dos profissionais de suporte para tecnologia da informação. Assim, o Ministério da Saúde assume o compromisso de garanti-la, estabelecendo cooperação e parcerias no processo de inserção, incluindo cursos na modalidade de Educação à Distância (EAD) por meio do site da Universidade Aberta do Sistema Único de Saúde (UNA-SUS) ${ }^{16}$.

Com base na análise do discurso, constata-se que os profissionais realmente se apropriaram de todo o conhecimento necessário para manejar a nova ferramenta, e que foi de extrema importância a iniciativa do Ministério em atentar para essa necessidade, já que alguns profissionais não são aptos a operar inovações tecnológicas.

Entende-se, em vista disso, que a qualificação dos profissionais de saúde permite aprofundar a experiência e apropriar os conhecimentos e informações, para produzir impacto no aperfeiçoamento dos índices de saúde da população brasileira ${ }^{17}$.

Ainda na perspectiva da implantação, dez profissionais ACS abordaram questões acerca da constante preparação dos profissionais, proporcionando a construção do DSC D, que teve como ideia central a educação permanente dos ACS para a implantação do e-SUS. Para eles:

Foi e está sendo um processo de aprendizado contínuo e de transição. Foi uma coisa nova pra gente, lenta e de grande pressão para os profissionais, pois não estávamos preparados para preencher as fichas, os dados, todos contêm muitas informações, foi muito complicado. Trouxe inovações e, por isso, no começo a gente fica um pouco preocupado, mas, aos poucos, tudo foi se encaixando, já que estamos sempre preparados para os processos de mudanças. (DSC D)

É notória a preocupação dos ACS com as mudanças e a necessidade da sua participação, nos processos formativos, para fornecer segurança na prática do novo, tendo em vista que todas as ideias de mudança na reestruturação do sistema só serão cumpridas e efetivas se houver envolvimento e dedicação dos gestores, dos profissionais de saúde e dos trabalhadores do SUS na implantação, na utilização e no aprimoramento do Sisab e da estratégia e-SUS AB. Posto que se trata de um processo contínuo, que exige tempo e muita sapiência para que possa ser, na prática, equivalente a tudo aquilo que foi idealizado na teoria revelada na literatura sobre o assunto, é preciso que esse envolvimento e dedicação se traduzam em adaptá-lo, da melhor forma possível, a cada cenário de um território tão diversificado como o do Brasil ${ }^{18}$.

No que se refere à relevância do gerente da unidade básica de saúde para o processo de incorporação do e-SUS, as respostas dos seis enfermeiros gerentes proporcionaram a construção do DSC E, que teve como ideia central a atividade de gerenciamento na implantação do e-SUS. Conforme relataram: 
Foi bastante intensa; realmente, capacitar a equipe para que ela identifique as dificuldades e já tente consertar o que for necessário fazendo link e tirando dúvidas, com o apoio da Secretaria de Saúde, levantadas pelos profissionais com base no que estariam vivenciando e outras que iam surgindo. Todo o mundo se envolveu do mesmo jeito. Servimos como base apoiadora para os demais profissionais, principalmente para os ACS, nos casos de alguma dúvida ou necessidade. O gerenciamento é de extrema importância, pois tem o objetivo de monitorar cada categoria nas produções, principalmente as categorias de ACS e digitadores, para entregarem em dia, semanalmente, mas também os demais membros da equipe. (DSC E)

A atuação do enfermeiro é primordial para que se alcancem os objetivos esperados na execução das estratégias do programa voltado para a saúde da família, bem como para o e-SUS, sendo esse profissional reconhecido por sua atuação gerencial junto à equipe de saúde, pois todas as ações têm melhor desempenho quando têm a garantia da sua participação. No e-SUS, o papel gerencial do enfermeiro garante não só o sucesso das ações esperadas com o uso dessa estratégia, mas também o monitoramento de possíveis limitações e dificuldades, de modo a solucioná-las. As funções gerenciais, apontadas como encargo do enfermeiro, permitem compreender com maior nitidez que "gerenciar" é uma ferramenta que faz parte do processo do trabalho de "cuidar", que tem como pressuposto final a atenção à saúde ${ }^{19-20}$.

É possível identificar no discurso o empenho dos enfermeiros gerentes em administrar todo o processo de inserção do novo sistema, assim como do restante da equipe rotineiramente. Esse fato é de extrema relevância para atingir as metas propostas pelo Ministério da Saúde em relação ao programa e à própria população.

Ao indagar a respeito dos fatores facilitadores da implantação do e-SUS, quatro profissionais ACS salientaram o benefício de melhor conhecer e acompanhar a população, o que proporcionou a construção do DSC F, que tem como ideia central os aspectos positivos do e-SUS no processo de trabalho dos ACS. Segundo eles:

Vejo como positivo o empoderamento da inserção das famílias no sistema, contribuindo assim para melhor acompanhamento, pois na ficha há muitas perguntas que, antes, a gente não tinha interesse em saber; agora. Precisamos saber e, além disso, as informações vêm mais esclarecidas, mais organizadas para que possam melhorar o trabalho. (DSC F)

A partir do DSC F, ficam evidenciados os pontos positivos e facilitadores do processo de trabalho, fornecidos por essa estratégia, por meio da qual, segundo afirmam os ACS, é possível ter mais controle do acompanhamento feito aos usuários, que antes não era visto com tanta importância.

Para facilitar ainda mais o acesso às informações, o Ministério da Saúde possibilitará, por meio do prontuário eletrônico, a visualização na folha de rosto dos dados registrados na primeira consulta, durante o atendimento do cidadão. Assim, as informações sobre o motivo de busca do serviço de saúde e dados vitais avaliados na escuta inicial poderão ser consultados pelo profissional que fizer o atendimento ${ }^{10}$.

No que concerne às dificuldades de implantação do e-SUS, observa-se que as respostas de 16 ACS proporcionaram a construção do DSC G, que tem como ideia central os aspectos restritivos do e-SUS no processo de trabalho dos ACS, que afirmam:

Uma das grandes dificuldades foi a falta de internet pela qual passamos durante seis meses. Também é complicada a aceitação da comunidade no que se refere a fornecer tantas informações. É notória a resistência inicial, e é preciso uma árdua adaptação das pessoas envolvidas no processo para convencer alguns usuários a cederem as informações solicitadas no cadastro, pois alguns não compreendem o 
objetivo do cadastramento. O programa exige dados mais completos e, então, para obtê-los, necessitamos de acesso aos documentos da população, como RG e CPF. (DSC G)

Essa resistência da população por falta de conhecimento sobre o programa tem dificultado o processo de cadastramento, e essa tem sido uma das grandes reclamações dos profissionais, que demoram mais para concluir os cadastros ou, até mesmo, não o fazem por falta das informações necessárias não fornecidas. Na prática da visita domiciliar, ao chegarem nas residências, os ACS enfrentam a problemática de não serem recebidos pelas famílias, as dificuldades de encontrar o usuário em sua residência, os portões fechados, sobretudo moradores que relatam não quererem ser incomodados por esses agentes. Segundo as famílias, essa rejeição ocorre normalmente porque, no momento da visita domiciliar não necessitam de seus cuidados, o que revela o desconhecimento das atribuições desse profissional e da ESF, levando à desvalorização desse trabalho, em decorrência das compreensões equivocadas ${ }^{21}$.

A implantação do e-SUS AB configura-se como um importante progresso na qualificação e no uso da informatização durante as ações de saúde desenvolvidas na atenção básica. Como em todo momento de transformações, há um período inicial de adaptação que é mais instável até que os novos fluxos e instrumentos utilizados sejam inseridos na rotina dos profissionais das equipes de saúde.

A informação dispõe de um papel planejado a ser desempenhado nos processos de tomadas de decisão; todavia, ainda é possível constatar, por um lado, a presença de inúmeras dificuldades para acessar e tratar os dados de forma adequada, de modo a dispor de informações necessárias ao processo de trabalho em saúde, em momento cabível; por outro, a ausência de conexão entre os processos de planejamento e de gestão da saúde e os próprios sistemas de informação ${ }^{22}$.

Assim, a produção e disseminação de informação e dados relativos à saúde precisa atender tanto às necessidades dos usuários, quanto às dos profissionais e gestores. Neste sentido, é fundamental a existência de equipamentos que garantam a funcionalidade e agilidade dos sistemas, como por exemplo, computadores, impressoras e acesso à internet ${ }^{23}$.

\section{Conclusão}

De modo geral, as entrevistas revelaram que os profissionais compreendem que, além de um sistema de informação para organizar e armazenar dados, o e-SUS é uma importante ferramenta para auxiliar na gestão do cuidado na atenção básica à saúde. Todavia, ainda existem aspectos restritivos para o seu funcionamento, tais como a escassez de recursos e a falta de adesão da população.

Nesse sentido, o desenvolvimento da pesquisa, ora apresentada, a partir das vivências e relatos de profissionais permitiu constatar que o e-SUS colabora com o sistema de saúde, uma vez que atende às finalidades preconizadas pelo Ministério da Saúde. No entanto, ainda persistem algumas dificuldades no que diz respeito à adaptação a um novo sistema.

Nessa lógica, julga-se importante que os gestores atentem para as concepções e convicções dos profissionais das equipes de saúde acerca do e-SUS, com o intuito de constatar os avanços a serem realizados para assegurar a fidedignidade dos dados coletados e informações produzidas, de maneira a garantir a qualidade da informação para subsidiar as intervenções de saúde e as tomadas de decisão.

Cabe ponderar, ainda, que foram identificadas algumas limitações no desenvolvimento do estudo, tais como as dificuldades de acesso aos profissionais da saúde. Desse modo, sugere-se a ampliação do estudo para a incorporação das narrativas de gestores e usuários, de forma a explorar um maior número de concepções a respeito do tema estudado.

Esse estudo foi realizado com o intuito não só de evidenciar o processo de implantação do e-SUS AB, mas também de contribuir para a ampliação de pesquisas ligadas a essa temática e para tornar mais efetivo 
e ágil o processo de trabalho dos profissionais da saúde concatenado com as ferramentas tecnológicas, visto que o conteúdo dessa ferramenta ainda se faz pouco exposto na literatura por dizer respeito a um sistema de informação recentemente criado e utilizado.

\section{Referências}

1. Ministério da Saúde (BR). $8^{\circ}$ Conferência Nacional de Saúde: relatório final [Internet]. Brasília: 1986 [citado em 2017 out. 5]. Disponível em: https://goo.gl/Eh4Qas

2. Westphal MF, Eurivaldo SA. Gestão de serviços de saúde: descentralização, municipalização do SUS. São Paulo: Edusp; 2001.

3. Presidência da República (BR). Casa Civil. Subchefia de Assuntos Jurídicos. Lei no 8.080, de 19 de setembro de 1990. Dispõe sobre as condições para a promoção, proteção e recuperação da saúde, a organização e o funcionamento dos serviços correspondentes, e dá outras providências. DOU [Internet]. 1990 [citado em 2017 out. 5]. Disponível em: https://goo.gl/1DJG5j

4. Gamarra J, Salvador J. Controle social na saúde: desafios e perspectivas. In: Machado, HB; Leandro, JA; Michaliszyn, MS, organizadores. Saúde coletiva: um campo em construção. Curitiba: Ibepex; 2006. p. 343.

5. Presidência da República (BR). Casa Civil. Subchefia de Assuntos Jurídicos. Decreto n 7.530, de 21 de julho de 2011. Aprova a estrutura regimental e o quadro demonstrativo dos cargos em comissão e das funções gratificadas do Ministério da Saúde. DOU [Internet]. 2011 [citado em 2017 set. 5]. Disponível em: https://goo.gl/BxrLfi

6. Ministério da Saúde (BR). Portaria n 2.488, de 21 de outubro de 2011. Aprova a Política Nacional de Atenção Básica, estabelecendo a revisão de diretrizes e normas para a organização da Atenção Básica, para a Estratégia Saúde da Família (ESF) e o Programa de Agentes Comunitários de Saúde (PACS). DOU [Internet]. 2011 [citado em 2018 jan. 5]. Disponível em: http://bvsms.saude.gov.br/bvs/saudelegis/ gm/2011/prt2488 $21 \quad 10$ 2011.html

7. Ministério da Saúde (BR). Política Nacional de Atenção Básica [Internet]. Brasília; 2006 [citado em 2018 jan. 5]. (Série E. Legislação em Saúde). Disponível em: http://189.28.128.100/dab/docs/publicacoes/ geral/pnab.pdf

8. Ministério da Saúde (BR). e-SUS Atenção Básica: sistema com coleta de dados simplificada: CDS. Brasília; 2013 [citado em 2018 jan. 5]. Disponível em: http://189.28.128.100/dab/docs/portaldab/ documentos/manual cds.pdf

9. Prefeitura Municipal de Sobral (CE). Vigilância epidemiológica. Dados da atenção básica à saúde. Sobral: Editora da Secretaria de Saúde; 2015.

10. Lefèvre F, Lefèvre AMC, Cavalcanti MA, Teixeira VJJ. O discurso do sujeito coletivo: uma nova abordagem metodológica em pesquisa qualitativa. Caxias do Sul: Educs; 2000.

11. Ministério da Saúde (BR). Conselho Nacional de Saúde. Resolução n. 466, de 12 de dezembro de 2012. Aprova as diretrizes e normas regulamentadoras de pesquisas envolvendo seres humanos. DOU [Internet]. 2012 [citado em 2018 set. 16]. Disponível em http://bvsms.saude.gov.br/bvs/saudelegis/ cns/2013/res0466 $12 \quad 12 \quad 2012 . h t m l$

12. Who Global Observatory For Ehealth. Building foundations for eHealth: progress of member states: report of the Global Observatory for eHealth [Internet]. Geneva; 2006 [citado em 2018 jan. 5]. Disponível em: http://apps.who.int/iris/handle/10665/43599

13. Ministério da Saúde (BR); Organização Pan-Americana da Saúde; Fundação Oswaldo Cruz. A experiência brasileira em sistemas de informação em saúde. Vol. 2, Falando sobre os sistemas de informação em saúde no Brasil. Brasília; 2009 [citado em 2018 jan. 5]. (Série B. Textos Básicos de Saúde). Disponível em: https://goo.gl/KYFrSY

14. Marin HF. Sistemas de informação em saúde: considerações gerais. J Health Inform [Internet]. 2010 [citado em 2017 dez. 5];2(1):20-4. Disponível em: https://goo.gl/EGVjCU

15. Cavalcante RB, Cunha SGS, Bernardes MFVG, Gontijo TL, Guimarães EAA, Oliveira VC. Sistema de Informação Hospitalar: utilização no processo decisório. ] Health Inform [Internet]. 2012 [citado em 2017 maio 18];4(3):73-9. Disponível em: http://www.jhi-sbis.saude.ws/ojs-jhi/index.php/jhi-sbis/article/ view/199/120 
16. Conselho Nacional de Secretaria da Saúde (BR). Estratégia e-SUS Atenção Básica e Sistema de Informação em Saúde da Atenção Básica - SISAB [Internet]. Brasília, 2013 [citado em 2017 dez. 5]. (Coleção Progestores. Nota técnica 07). Disponível em: http://www.conass.org.br/biblioteca/wp-content/ uploads/2013/01/NT-07-2013-e-SUS-e-SISAB.pdf

17. Rangel-S ML, Barbosa AO, Riccio NCR, Souza JS. Redes de aprendizagem colaborativa: contribuição da educação a distância no processo de qualificação de gestores do Sistema Único de Saúde - SUS. Interface (Botucatu) [Internet]. 2012 jul. [citado em 2017 maio 18];16(41):545-56. doi: http://dx.doi. org/10.1590/S1414-32832012005000031

18. Mascarenhas $\mathrm{AO}$, Vasconcelos $\mathrm{CF}$, Vasconcelos IFG. Impactos da tecnologia na gestão de pessoas: um estudo de caso. R Adm Contemp [Internet]. 2005 mar. [citado em 2017 maio 18]; 9(1):125-47. doi: http://dx.doi.org/10.1590/S1415-65552005000100007

19. Nascimento IJ, Leitão Rosa ER, Vargens OM. A qualidade nos serviços de saúde pública segundo enfermeiros que gerenciam unidades básicas de saúde. Rev Enferm [Internet]. 2006 set. [citado em 2017 maio 18];14(3):350-6. Disponível em: https://goo.gl/7RSFGG

20. Aguiar ABA, Costa BSR, Weirich FC, Bezerra QLA. Gerência dos serviços de enfermagem: um estudo bibliográfico. R Eletron Enferm [Internet]. 2005 dez. [citado em 2017 maio 18];07(3):318-26. doi: http://dx.doi.org/10.5216/ree.v7i3.901

21. Santana JCB, Fortes NM, Gonçalves CP, Moura IC, Porto PA. Visita domiciliar dos agentes comunitários de saúde no planejamento das ações das estratégias da saúde da família: avanços e desafios. Enferm R [Internet]. 2015 ago. [citado em 2017 maio 18];18(2):18-28. Disponível em: http://periodicos. pucminas.br/index.php/enfermagemrevista/article/view/11691

22. Gava M, Ferreira LS, Palhares D, Mota ELA. Incorporação da tecnologia da informação na Atenção Básica do SUS no Nordeste do Brasil: expectativas e experiências. Ciênc. saúde coletiva [Internet]. 2016 jul. [citado em 2017 dez. 5];21(3):891-902. doi: http://dx.doi.org/10.1590/1413-81232015213.01062015

23. Andrade CCR. Equipamentos de informatização nas unidades de atenção básica do Brasil: análise baseada no programa nacional de melhoria do acesso e da qualidade - PMAQ [tese na Internet]. Rio Grande do Sul: Universidade Federal do Rio Grande do Sul; 2014 [citado em 2017 dez. 5]. Disponível em: https://goo.gl/eowKNB 